\title{
Cardiovascular disease and HIV infection
}

\author{
Magdalena Jachymek ${ }^{1}$, Marta Braksator ${ }^{1}$, Miłosz Parczewski ${ }^{2}$, Małgorzata Peregud-Pogorzelska ${ }^{1}$, \\ Jarosław Kaźmierczak ${ }^{1}$ \\ ${ }^{1}$ Department of Cardiology, Pomeranian Medical University in Szczecin, Poland \\ ${ }^{2}$ Department of Infectious, Tropical Diseases and Immune Deficiency, Pomeranian Medical University in Szczecin, Poland
}

\begin{abstract}
Advances in antiretroviral therapy have resulted in a significant increase in life expectancy and quality of life of people living with human immunodeficiency virus (HIV) (PLWH). However, long-term observation of this population revealed an increased risk of cardiovascular diseases (CVDs). Moreover, development of atherosclerosis may be secondary to numerous factors. Traditional risk factors of ischemic heart disease, such as hypertension, diabetes, and cigarette smoking, are more common in HIV-infected population than in non-HIV-infected one. Many antiretroviral drugs have an unfavorable metabolic profile, leading to dyslipidemia, lipodystrophy, and impaired glucose metabolism. Markers of immuno-activation, coagulation, and endothelium dysfunction, may remain elevated despite an effective antiretroviral treatment (ART). Inflammation affects arterial endothelium, leading to an increased deposition of lipids in the arterial wall. HIV infection may also affect blood pro-thrombotic activity. All these factors lead to more rapid atherosclerosis formation and increased risk of myocardial infarction. In order to lower cardiovascular risk in PLWH, traditional risk factors should be modified, and ART with less impact on patients' metabolisms should be used. If necessary, a lipid-lowering treatment should be introduced. Treatment with statins brings an additional benefit of reducing inflammatory markers associated with an increased CVD risk. When selecting a statin, possible interactions with ART need to be considered.
\end{abstract}

HIV AIDS Rev 2021; 20, 2: 85-89 DOI: https://doi.org/10.5114/hivar.2021.107234

Key words: AIDS, HIV, cardiovascular disease, ischemic heart disease.

\section{Introduction}

As a result of effective antiretroviral treatment, over $90 \%$ of people living with human immunodeficiency virus (PLWH) achieve an undetectable HIV viral load, which contributes to the reduction of disease spread and progression [1]. Life expectancy of HIV-infected patients is now comparable to that in the general population. A successful antiretroviral treatment (ART) has reduced the number of deaths associated with opportunistic infections; however, a higher risk of cardiovascular disease (CVD) remains an open issue. Recent meta-analyses showed that a chronic HIV infection

was associated with a more than doubled risk of myocardial infarction [2] and cardiovascular disease [3].

The pathophysiology of CVD in the population of PLWH is complex, and results from the infection itself, impairment of the immune system, adverse effects of ART drugs, and a widespread distribution of traditional risk factors among PLWH. The relative risk of myocardial infarction in the infected population compared to the uninfected population is 1.53 (95\% CI: $1.32-1.75 \%$ ), and remains significant even after considering traditional risk factors [4]. Coronary computed tomography (CCT) of coronary vessels with measurement of coronary calcium score (CCS) indicate over three times higher risk of development of the non-calcified athe-

Article history:

Received: 03.08.2020

Received in revised form: 09.09.2020

Accepted: 28.09.2020

Available online: 30.06 .2021
International Journal of HIV-Related Problems

HIV \& AIDS

R e vi e w 
rosclerotic plaque [5]. Also, a significant increase of intima media thickness (IMT) of the carotid artery, an indicator of sub-clinical atherosclerosis, is observed in comparison to healthy population $[6,7]$.

The aim of this paper was to summarize risk factors of CVD in the population of PLWH. Possible management of dyslipidemias will be discussed as well as invasive treatment possibilities.

\section{Traditional risk factors of ischemic heart disease in HIV-infected population}

Epidemiological studies indicated that traditional risk factors of ischemic heart disease (IHD) occur more often in PLWH population. In a large cohort study involving 3,851 people infected with HIV, with a control group of $1,044,589$ healthy individuals, a significantly more common incidence of arterial hypertension ( $21.2 \%$ vs. $15.9 \%)$, diabetes ( $11.5 \%$ vs. $6.6 \%)$, and dyslipidemia (23.3\% vs. $17.6 \%$ ) were observed [4].

As compared to sex-, age-, and race-matched control group, smoking is significantly more common in patients infected with HIV ( $54 \%$ vs. $44 \%$ of ever smokers, $42 \%$ vs. $30 \%$ of active smokers, both with $p<0.001)$. Moreover, PLWH show a lower probability of breaking the habit [8].

\section{Effects of antiretroviral treatment on metabolic profile}

As any other treatment, ART is not adverse effects-free. The therapy affects metabolic profile, causing dyslipidemia, insulin resistance, glucose tolerance disorders, and lipodystrophy syndrome.

HIV patients treated with ART have a 52\% higher risk of IHD compared to people who did not receive the treatment, while people treated with protease inhibitors (PIs) demonstrate a $41 \%$ higher risk compared to patients treated using other schemes [9]. PIs (especially lopinavir and ritonavir) increase the hepatic production of VLDL, and reduce the activity of lipoprotein lipase, therefore increasing the triglyceride level and, to a lesser extent, the blood total cholesterol level as well as decreasing the level of HDL cholesterol. Dyslipidemia may be caused by a similarity between the catalytic region of HIV-1 protease and two proteins participating in the metabolism of lipids, including CRABP-1 (cytoplasmic retinoic acid-binding protein type 1) and LRP1 (low density lipoprotein receptor-related protein type 1). LRP1 is a protein binding to lipoprotein lipase that decomposes triglycerides, being the main component of VLDL, allowing free fatty acids to penetrate into the adipose tissue. The hypolipidemic effect of PIs is also related to the polymorphism of the APOCIII gene and reduced activity of antioxidative enzymes [10]. Patients undergoing ART present a different structure of lipoproteins, compared to healthy population. Those differences are best visible in HDLs, which are bigger and less stable [11].

Lipodystrophy, defined as a reduction of the subcutaneous adipose tissue in the extremities, face, and buttocks, and accumulation of the visceral adipose tissue, is associated with a therapeutic use of PIs and nucleoside reverse transcriptase inhibitors (NRTIs). PIs inhibit the function of CRABP-1 protein and lead to increased apoptosis and reduced differentiation of adipocytes, especially in peripheral tissues, which consequently leads to lipoatrophy. The visceral adipose tissue seems to be spared from that process, in turn leading to increased insulin resistance [10].

NRTIs cause mitochondrial dysfunction. As analogues of nucleotides, NRTIs are incorporated into mitochondrial DNA (mtDNA) by mitochondrial polymerase gamma. This precedes to the formation of mtDNA chains containing nucleotide analogues, which may result in early termination of translation. Disturbances in the process of mitochondrial proteins production, result in an increased production of free radicals, oxidized forms of proteins and lipids. Reduced levels of mRNA for selected mitochondrial proteins were observed in patients with lipodystrophy, e.g., lipoprotein lipase and the glucose transporter GLUT4. On the other hand, a higher level of mRNA for transporters of free fatty acids and IL6 was noted. Excessive production of free radicals directs cells towards apoptosis [12].

Glucose tolerance disorders associated with insulin resistance, predispose patients undergoing ART to development of type 2 diabetes. The relative risk may be even four times higher compared to the healthy population $(\mathrm{RR}=4.11$; CI 95\%: $1.85-9.16 \%$, adjusted for age and BMI) [13]. Similarly to lipodystrophy, diabetes is mostly associated with the treatment with PIs and NRTIs [14, 15]. Patients with a lower baseline CD4+ cells count show a higher risk of developing insulin resistance after introduction of ART [16].

\section{Dysfunction of the immune system and endothelium}

Dysfunction of the immune system is considered to be an additional risk factor of coronary disease in the population infected with HIV. Residual immune dysregulation syndrome (RIDS), the state of increased immuno-activation despite suppression of the virus replication, is observed in patients treated with ART. The syndrome manifests itself with increased levels of inflammatory markers, such as hsCRP, IL-6, and D-DIMER compared to the healthy population [17].

Lipoproteins, lymphocytes, macrophages, and the endothelium participate in a formation of atherosclerotic plaque. Infection with HIV causes a dysfunction of lymphocytes and macrophages, an increased production of proinflammatory cytokines, such as IL-6 and IL-18 [18], which affects the number of receptors responsible for binding macrophages to endothelial cells and impairs the intracellular lipid metabolism. Monocytes in the blood of PLWH produce more IL- $1 \beta$, which stimulates the production of IL- 8 and IL-6 [19]. Increased levels of IL-6, D-DIMER, and hsCRP are associated with a significantly higher risk of death due to cardiovascular reasons (hazard ratio - HR: 1.72, 1.73, and 1.44 , respectively; $p<0.05$ ) [20]. 
It was demonstrated that there was a higher expansion of non-classic monocytes (CD14+, CD16++) in the blood of PLWH. Those monocytes were characterized by higher proinflammatory activity, containing higher level of the MCP1 protein (increasing diapedesis of monocytes to the intima), which results in an increased migration of monocytes into the atherosclerotic plaque, which was associated with a more rapid progression of atherosclerosis in CCT [21]. A higher count of CD14+/CD16+ monocytes is correlated with a higher concentrations of IL-6 and hsCRP [22].

A chronically increased count and activity of CD8 cells, the level of IL 6, tumor necrosis factor alpha (TNF), and fibrinogen in the PLWH population, positively associate with the IMT [23].

A correlation was also demonstrated between increased levels of D-DIMER, hsCRP, sCD-14, IL-6, and microcirculation disorders caused by an endothelial dysfunction, which may also play a role in heart ischemia in that population $[24,25]$.

The HIV-associated immuno-activation may also be caused by disruption of the intestinal barrier, which may result in increased translocation of bacteria to the blood. The soluble CD-14 (sCD-14) and MD-2, proteins responsible for binding to the lipopolysaccharide (LPS) of the bacterial wall, are markers of bacterial translocation. The soluble CD-14 is an independent risk factor of death in the course of HIV infection [26].

PLWH have a higher level of oxLDL, a proinflammatory, oxidized form of LDL. Oxidized LDLs play a key role in formation of the atherosclerotic plaque. OxLDL is an independent risk factor of IHD in that group of patients [27].

\section{Increased pro-thrombotic activity}

An HIV infection may affect the blood pro-thrombotic activity. SMART study focused on the effect of untreated HIV infection on the concentration of extrinsic pathway coagulation factors. Patients with an uncontrolled replication of HIV, who did not undergo antiretroviral treatment, showed a higher activity of factor VIII and von Willebrand factor as well as lower activity of factors II, VII, IX, X, antithrombin, and $\mathrm{C}$ and $\mathrm{S}$ proteins (mostly produced in the liver). Thrombin generation ability was estimated using validated computational modelling. A higher thrombin generation ability and thus, increased coagulation potential, were demonstrated in the group of patients not undergoing antiretroviral therapy. It was noted that it was the changes in factor VIII and antithrombin III concentrations that were of key importance, suggesting that coagulation abnormalities might be caused by changes in the hepatocyte function [28].

Several markers associated with the dysfunction of coagulation system, indicating an increased risk of myocardial infarction, were identified. One of them is soluble urokinase plasminogen activator receptor (suPAR), which inhibits fibrinolysis by binding plasminogen urokinase activator. In the HIV-infected patients' population, an increased suPAR level occurs several years before the first acute coronary event, and it is a strong marker of the myocardial infarction risk in that population [29].

Another marker of elevated risk of myocardial infarction is plasminogen activator inhibitor 1 (PAI-1), a fibrinolysis activator produced by endothelial cells. An increase in the PAI- 1 level by $1 \mathrm{ng} / \mathrm{ml}$ (12 months before an acute coronary event) indicates a $1 \%$ increase in the risk of myocardial infarction [30].

\section{Reducing cardiovascular disease risk}

If necessary, all traditional IHD risk factors need to be modified. Lifestyle changes, which are usually recommended in IHD (diet, exercise, smoking cessation, etc.), are effective in reducing the cardiovascular risk in the PLWH population [31]. Exercise interventions are difficult due to a poor cardiorespiratory fitness level, which is additionally reversely correlated with inflammatory markers [32].

Recent ESC guidelines on the management of dyslipidemia published in 2019, emphasize the importance of lipid control, especially in patients with a very high and high cardiovascular risk. And, HIV-infected patients are considered to be exposed to a high cardiovascular risk [33].

PLWH have to be regularly screened to detect metabolic disorders caused by ART. In the case of confirmed dyslipidemia associated with the introduction of ART, changing ART drugs to those with a more favorable metabolic profile may be an option; however, an assessment of detailed changes in antiretroviral therapy is beyond the scope of this review.

If optimizing antiretroviral treatment proves ineffective or impossible to apply, introduction of a lipid-lowering agent may be necessary. Statins are the basic drugs used to reduce total cholesterol and LDL cholesterol levels. The mechanism of statin activity relies on inhibiting hepatic HMG-CoA reductase, and the effectiveness of statins was confirmed in CCT (reduction of non-calcified atherosclerotic plaque) [34]. Potential interactions with antiretroviral drugs have to be considered when selecting a statin. Most significant interactions are observed between PIs and simvastatin, and lovastatin and atorvastatin. PIs inhibit hepatic CYP3a4 cytochrome that is responsible for the metabolism of statins [35].

As statins exhibit a pleiotropic effect, it is suggested that some additional effects related to reducing inflammation may occur. A randomized clinical trial SATURN HIV evaluated effects of $10 \mathrm{mg}$ rosuvastatin on immune activation of HIV-positive patients with no dyslipidemia (baseline LDL $<130 \mathrm{mg} / \mathrm{dl}$ ). SATURN HIV study demonstrated a significant reduction of NTproBNP [36], activation of monocytes $[37,38]$, T cells CD 4+ and CD8+ count [38], and the oxLDL level [39]. Atorvastatin leads to a reduction of oxLDL, LDL, and total cholesterol levels, simultaneously reducing CTassessed volume of the atherosclerotic plaque [27].

Proprotein convertase subtilisin/ kexin 9 (PCSK9) inhibitors are new drugs used in IHD and hyperlipidemia to reduce LDL. PCSK9 levels in HIV-positive patients are elevated and relate to systemic markers of immuno-activation [40]. A ran- 
domized control trial designed to assess safety and efficacy of PCSK9 inhibitor (evolocumab) is in progress [41].

Omega-3 fatty acids have proven to successfully reduce the level of triglycerides, but not markers of inflammation in HIV-seropositive patients [42].

\section{Percutaneous coronary interventions}

The safety and efficacy of coronary angioplasty in HIV-infected patients are comparable to non-HIV-infected patients. If possible, implantation of a drug eluting stent (DES) is preferred, as it is associated with a reduced risk of another cardiac event [43]. The risk of restenosis is higher in people with persistent high levels of CRP and IL8 during 6-month post-intervention period [44]. Latest research shows that women are less likely to receive an appropriate treatment with cardiovascular procedures, than men [45].

\section{Conclusions}

Patients infected with HIV have a high cardiovascular risk. The pathophysiology of IHD in that population is complex and involves dysregulation of the immunological system, effects of drugs used for the antiretroviral therapy, and a high incidence of traditional risk factors. PIs and NRTIs may cause dyslipidemia, lipodystrophy syndrome, and glucose intolerance. Residual immune dysregulation syndrome (RIDS) is the state of increased immuno-activation, despite suppression of the virus replication, and is responsible for increased cardiovascular risk. Statins are the treatment of choice in case of dyslipidemia in this population due to some potential anti-inflammatory effect.

\section{Conflict of interest}

The authors declare no conflict of interest with respect to the research, authorship, and/or publication of this article.

\section{References}

1. Parczewski M, Siwak E, Leszczyszyn-Pynka M, et al. Meeting the WHO 90\% target: antiretroviral treatment efficacy in Poland is associated with baseline clinical patient characteristics: Antiretroviral. J Int AIDS Soc 2017; 20: 21847.

2. Rao SG, Galaviz KI, Gay HC, et al. Factors associated with excess myocardial infarction risk in HIV-infected adults: a systematic review and meta-analysis. J Acquir Immune Defic Syndr 2019; 81: 224-230.

3. Shah ASV, Stelzle D, Lee KK, et al. Global burden of atherosclerotic cardiovascular disease in people living with HIV. Circulation 2018; 138: 1100-1112.

4. Triant VA, Lee H, Hadigan C, Grinspoon SK. Increased acute myocardial infarction rates and cardiovascular risk factors among patients with human immunodeficiency virus disease. J Clin Endocrinol Metab 2007; 92: 2506-2512.

5. D’Ascenzo F, Cerrato E, Calcagno A, et al. High prevalence at computed coronary tomography of non-calcified plaques in asymptomatic HIV patients treated with HAART: a meta-analysis. Atherosclerosis 2015; 240: 197-204.
6. Stein JH, Currier JS, Hsue PY. Arterial disease in patients with human immunodeficiency virus infection: what has imaging taught us? JACC Cardiovasc Imaging 2014; 7: 515-525.

7. Hulten E, Mitchell J, Scally J, Gibbs B, Villines TC. HIV positivity, protease inhibitor exposure and subclinical atherosclerosis: a systematic review and meta-analysis of observational studies. Heart 2009; 95: 1826-1835.

8. Regan S, Meigs JB, Grinspoon SK, Triant VA. Determinants of smoking and quitting in HIV-infected individuals. PLoS One 2016; 11 : e0153103.

9. Islam FM, Wu J, Jansson J, Wilson DP. Relative risk of cardiovascular disease among people living with HIV: a systematic review and meta-analysis. HIV Med 2012; 13: 453-468.

10. da Cunha J, Maselli LMF, Bassi Stern AC, Spada C, Bydlowski SP. Impact of antiretroviral therapy on lipid metabolism of human immunodeficiency virus-infected patients: old and new drugs. World J Virol 2015; 4: 56-77.

11. Gillard BK, Raya JL, Ruiz-Esponda R, et al. Impaired lipoprotein processing in HIV patients on antiretroviral therapy. Arterioscler Thromb Vasc Biol 2013; 33: 1714-1721.

12. Gerschenson M, Brinkman K. Mitochondrial dysfunction in AIDS and its treatment. Mitochondrion 2004; 4: 763-777.

13. Brown TT. Antiretroviral therapy and the prevalence and incidence of diabetes mellitus in the multicenter AIDS cohort study. Arch Intern Med 2005; 165: 1179-1184.

14. Ledergerber B, Furrer H, Rickenbach M, et al. Factors associated with the incidence of type 2 diabetes mellitus in HIV-infected participants in the swiss HIV cohort study. Clin Infect Dis 2007; 45: 111-119.

15. De Wit S, Sabin CA, Weber R, et al. Incidence and risk factors for new-onset diabetes in HIV-infected patients. Diabetes Care 2008; 31: 1224-1229.

16. Krishnan S, Schouten J, Atkinson B, et al. Metabolic syndrome before and after initiation of antiretroviral therapy in treatment-naive HIV-infected individuals. J Acquir Immune Defic Syndr 2012; 61: 381-389.

17. Neuhaus J, Jacobs DR Jr, Baker JV, et al. Markers of inflammation, coagulation, and renal function are elevated in adults with HIV infection. J Infect Dis 2010; 201: 1788-1795.

18. Crowe SM, Westhorpe CLV, Mukhamedova N, Jaworowski A, Sviridov $\mathrm{D}$, Bukrinsky $\mathrm{M}$. The macrophage: the intersection between HIV infection and atherosclerosis. J Leukoc Biol 2010; 87: 589-598.

19. Jalbert E, Crawford TQ, D'Antoni ML, et al. IL-1B enriched monocytes mount massive IL-6 responses to common inflammatory triggers among chronically HIV-1 infected adults on stable antiretroviral therapy at risk for cardiovascular disease. PLoS One 2013; 8: e75500.

20. Nordell AD, McKenna M, Borges AH, Duprez D, Neuhaus J, Neaton JD. Severity of cardiovascular disease outcomes among patients with HIV is related to markers of inflammation and coagulation. J Am Heart Assoc 2014; 3: e000844.

21. Zungsontiporn N, Tello RR, Zhang G, et al. Non-classical monocytes and monocyte chemoattractant protein-1 (MCP-1) correlate with coronary artery calcium progression in chronically HIV-1 infected adults on stable antiretroviral therapy. PLoS One 2016; 11 : e0149143.

22. Wilson EMP, Singh A, Hullsiek KH, et al. Monocyte-activation phenotypes are associated with biomarkers of inflammation and coagulation in chronic HIV infection. J Infect Dis 2014; 210: 1396-1406.

23. Longenecker CT, Funderburg NT, Jiang Y, et al. Markers of inflammation and CD8 T-cell activation, but not monocyte activation, are associated with subclinical carotid artery disease in HIV-infected individuals. HIV Med 2013; 14: 385-390.

24. Sinha A, Ma Y, Scherzer R, et al. Role of T-cell dysfunction, inflammation, and coagulation in microvascular disease in HIV. J Am Heart Assoc 2016; 5: e004243. 
25. Hileman CO, Longenecker CT, Carman TL, et al. Elevated D-dimer is independently associated with endothelial dysfunction: a crosssectional study in HIV-infected adults on antiretroviral therapy. Antivir Ther 2012; 17: 1345-1349.

26. Sandler NG, Wand H, Roque A, et al. Plasma levels of soluble CD14 independently predict mortality in HIV infection. J Infect Dis 2011; 203: 780-790.

27. Nou E, Lu MT, Looby SE, et al. Serum oxidized low-density lipoprotein decreases in response to statin therapy and relates independently to reductions in coronary plaque in patients with HIV. AIDS 2016; 30: 583-590.

28. Baker JV, Brummel-Ziedins K, Neuhaus J, et al. HIV replication alters the composition of extrinsic pathway coagulation factors and increases thrombin generation. J Am Heart Assoc 2013; 2: e000264.

29. Rasmussen LH, Knudsen A, Katzenstein TL, et al. Soluble urokinase plasminogen activator receptor (suPAR) is a novel, independent predictive marker of myocardial infarction in HIV-1-infected patients: a nested case-control study. HIV Med 2016; 17: 350-357.

30. Knudsen A, Katzenstein TL, Benfield T, et al. Plasma plasminogen activator inhibitor-1 predicts myocardial infarction in HIV-1infected individuals. AIDS 2014; 28: 1171-1179.

31. Lima EMO, Gualandro DM, Yu PC, et al. Cardiovascular prevention in HIV patients: results from a successful interventionprogram. Atherosclerosis 2009; 204: 229-232.

32. Webel AR, Jenkins T, Vest M, et al. Cardiorespiratory fitness is associated with inflammation and physical activity in HIV+ adults. AIDS 2019; 33: 1023-1030.

33. Mach F, Baigent C, Catapano AL, et al. 2019 ESC/EAS Guidelines for the management of dyslipidaemias: lipid modification to reduce cardiovascular risk: the task force for the management of dyslipidaemias of the European Society of Cardiology (ESC) and European Atherosclerosis Society (EAS). Eur Heart J 2020; 41: 111-188.

34. Lo J, Lu MT, Ihenachor EJ, et al. Effects of statin therapy on coronary artery plaque volume and high-risk plaque morphology in HIV-infected patients with subclinical atherosclerosis: a randomised, double-blind, placebo-controlled trial. Lancet HIV 2015; 2: e52-e63.

35. Feinstein MJ, Achenbach CJ, Stone NJ, Lloyd-Jones DM. A systematic review of the usefulness of statin therapy in HIV-infected patients. Am J Cardiol 2015; 115: 1760-1766.

36. Dirajlal-Fargo S, Kinley B, Jiang Y, et al. Statin therapy decreases NT-proBNP in HIV: randomized placebo-controlled trial. AIDS 2015; 29: 313-321.

37. Funderburg NT, Jiang Y, Debanne SM, et al. Rosuvastatin treatment reduces markers of monocyte activation in HIV-infected subjects on antiretroviral therapy. Clin Infect Dis 2014; 58: 588-595.

38. Funderburg NT, Jiang Y, Debanne SM, et al. Rosuvastatin reduces vascular inflammation and T-cell and monocyte activation in HIVinfected subjects on antiretroviral therapy. J Acquir Immune Defic Syndr 2015; 68: 396-404.

39. Hileman CO, Turner R, Funderburg NT, Semba RD, McComsey GA Changes in oxidized lipids drive the improvement in monocyte activation and vascular disease after statin therapy in HIV. AIDS 2016; 30: 65-73

40. Zanni MV, Stone LA, Toribio M, et al. Proprotein convertase subtilisin/kexin 9 levels in relation to systemic immune activation and subclinical coronary plaque in HIV. Open Forum Infect Dis 2017; 4: ofx227.

41. Boccara F, Kumar P, Caramelli B, et al. Evolocumab treatment in patients with HIV and hypercholesterolemia/mixed dyslipidemia: BEIJERINCK study design and baseline characteristics. Am Heart J 2020; 220: 203-212.

42. Amador-Licona N, Díaz-Murillo TA, Gabriel-Ortiz G, et al. Omega 3 fatty acids supplementation and oxidative stress in HIV-seropositive patients. A clinical trial. PLoS One 2016; 11: e0151637.
43. Badr S, Minha S, Kitabata H, et al. Safety and long-term outcomes after percutaneous coronary intervention in patients with human immunodeficiency virus. Catheter Cardiovasc Interv 2015; 85: 192 198.

44. Schneider S, Spinner CD, Cassese S, et al. Association of increased $\mathrm{CD} 8+$ and persisting $\mathrm{C}$-reactive protein levels with restenosis in HIV patients after coronary stenting. AIDS 2016; 30: 1413-1421.

45. Grinspoon SK, Grunfeld C, Kotler DP, et al. State of the science conference initiative to decrease cardiovascular risk and increase quality of care for patients living with HIV/AIDS executive summary. Circulation 2008; 118: 198-210. 